NISSUNA UMANA INVESTIGAZIONE SI PUO DIMANDARE VERA SCIENZIA S'ESSA NON PASSA PER LE MATEMATICHE DIMOSTRAZIONI LEONARDO DA VINCI

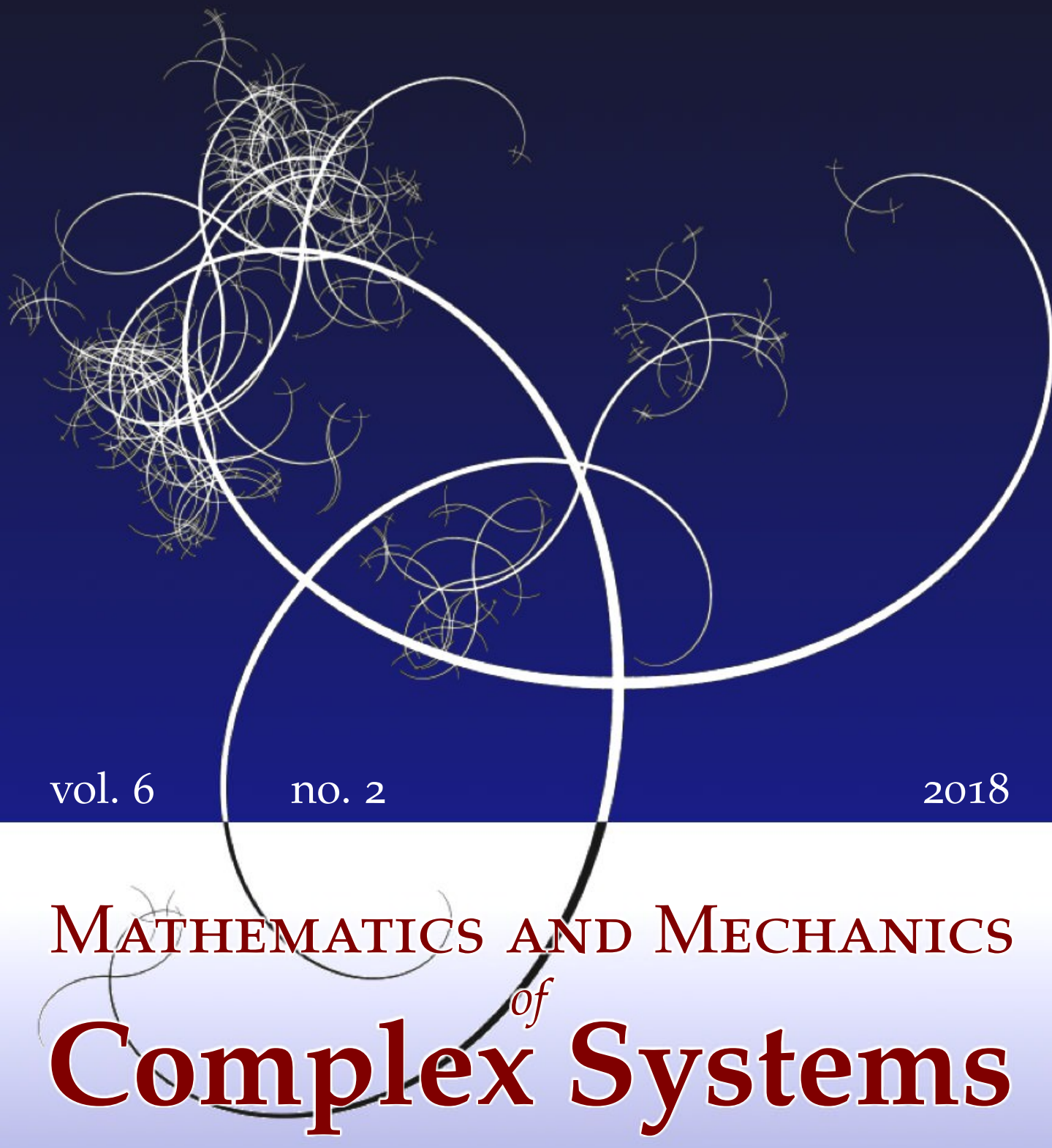

REUVEN SEGEV AND JĘDRZEJ ŚNIATYCKI

ON JETS, ALMOST SYMMETRIC TENSORS, AND TRACTION HYPER-STRESSES 


\title{
ON JETS, ALMOST SYMMETRIC TENSORS, AND TRACTION HYPER-STRESSES
}

\author{
REUVEn SEgEV AND JęDRZEJ ŚNIATYCKI
}

\begin{abstract}
This paper considers the formulation of higher-order continuum mechanics on differentiable manifolds devoid of any metric or parallelism structure. For generalized velocities modeled as sections of some vector bundle, a variational $k$-th order hyper-stress is an object that acts on jets of generalized velocities to produce power densities. The traction hyper-stress is introduced as an object that induces hyper-traction fields on the boundaries of subbodies. Additional aspects of multilinear algebra relevant to the analysis of these objects are reviewed.
\end{abstract}

\section{Introduction}

The present paper considers the basic mathematical objects in the analysis of hyperstresses for a theory defined on differentiable manifolds. Thus, generalized velocities are represented by sections of a vector bundle. Such a setting encompasses both the Lagrangian and Eulerian points of view of continuum mechanics as well as classical field theories of physics. The base manifold of the vector bundle is interpreted accordingly as either the body manifold, the physical space, or spacetime, respectively. It is recalled that as early as 1957, Walter Noll [1959] defined a body as a differentiable manifold. Further motivation for the general geometric setting of a manifold can be traced back to [Truesdell and Toupin 1960, p.660] (see also [Segev 1994; 2000; 2013]).

As a generalization of the standard introduction of hyper-stresses in higher-order continuum mechanics, the $k$-th order hyper-stress object, the variational hyperstress, is dual to $k$-jets of sections of the vector bundle (see [Segev 2017]). Continuum mechanics on manifolds differs from standard formulations in Euclidean spaces in the following significant sense. In traditional continuum mechanics, the

\section{Communicated by Francesco dell'Isola.}

Both authors are grateful to BIRS for sponsoring the Banff Workshop on Material Evolution, June 11-18, 2017, which lead to this collaboration. Segev's work has been partially supported by the H. Greenhill Chair for Theoretical and Applied Mechanics and the Pearlstone Center for Aeronautical Engineering Studies at Ben-Gurion University.

MSC2010: primary 74A10; secondary 53Z05.

Keywords: high-order continuum mechanics, differential geometry, vector bundle, hyper-stress, traction, multilinear algebra. 
stress tensor plays two roles: it acts on the derivatives of velocity fields to produce power densities and it induces traction fields on boundaries of subbodies. For a theory on manifolds, however, two distinct mathematical objects play these two roles (see [Segev 2002; 2013]). The variational stress acts on the jets of generalized velocity fields to produce power, while the traction stress induces the traction fields on the boundaries of subbodies. While the variational hyper-stress fields have been considered in [Segev 1986; 2017], we propose here a suitable candidate for the role of traction hyper-stress.

This paper is meant to be used as an introduction to the subject, and additional details regarding the properties of symmetric tensors, used extensively in the analysis of jets, are provided in the Appendix. Thus, Section 2 introduces the basic structure, motivates the use of jets of vector fields, and describes their very basic properties. Section 3 introduces variational hyper-stresses and Section 4 introduces traction hyper-stresses and describes the basic properties of what we refer to as "almost symmetric tensors" used to represent them locally. Finally, a short summary is given in Section 5.

\section{Jets}

Jet bundles, see [Saunders 1989] for a comprehensive treatment, serve as the fundamental objects in the formulation of higher-order continuum mechanics on differentiable manifolds. In this section we review the basic constructions associated with jet bundles of a vector bundle. Firstly, however, we motivate the use of jet bundles in higher-order continuum mechanics and classical field theories.

2.1. The fundamental structure. The basic object we consider here is a vector bundle:

$$
\pi: W \rightarrow \mathscr{X}
$$

The object $\mathscr{X}$ is assumed to be a smooth manifold of dimension $n$, that might have a boundary. We will refer to $\mathscr{X}$ as the base manifold. In the context of the Lagrangian point of view of continuum mechanics, $\mathscr{X}$ is interpreted as the body manifold. In the Eulerian point of view of continuum mechanics, $\mathscr{X}$ is interpreted as the physical space manifold, and in modern formulations of classical field theories, $\mathscr{X}$ is interpreted as the space-time manifold.

No additional structure, such as a Riemannian metric, a connection, a parallelism structure, is assumed for the base manifold. This level of generality is in accordance with the reluctance of modern presentations to use a preferred class of reference states (e.g., [Noll 1959]). In particular, if one wishes to consider live tissues in biomechanical studies, it is unlikely that a preferred reference state of the tissue may be pointed out. Thus, there is no class of preferred coordinate systems on $\mathscr{X}$ 
and denoting coordinates by $x^{i}, i=1, \ldots, n$, a coordinate transformation will be denoted by $x^{i^{\prime}}=x^{i^{\prime}}\left(x^{i}\right)$.

Tangent vectors to the manifold $\mathscr{X}$ are viewed as derivatives of the curves $c$ : $[a, b] \rightarrow \mathscr{X}$. (See [Abraham et al. 1988, pp. 157-158 and 479] for the definition of derivative of a curve at the endpoints and its application in the definition of the tangent space to a manifold at a boundary point.) The tangent space to $\mathscr{X}$ at $x$, denoted by $T_{x} \mathscr{X}$, contains all the tangent vectors at $x$ and the tangent bundle $T \mathscr{X}$ is the collection of all tangent vectors at the various points. Given a coordinate system $\left(x^{i}\right)$ and a point $x_{0}$ with coordinates $x_{0}^{j}$, one has coordinate lines, curves of the form $c_{i}:[a, b] \rightarrow \mathscr{X}$, such that their coordinate representation satisfy

$$
x^{j}(t)=x^{j}\left(c_{i}(t)\right)= \begin{cases}x_{0}^{j}, & \text { if } i \neq j, \\ x_{0}^{j}+t, & \text { if } i=j .\end{cases}
$$

The time derivatives of these curves induce tangent vectors denoted by $\partial_{i}=\dot{c}_{i}$. At each point $x$, the vectors $\left\{\partial_{i}\right\}, i=1, \ldots, n$, form a basis of $T_{x} \mathscr{X}$. The corresponding dual basis of the dual vector space, $T_{x}^{* \mathscr{X}}$, is denoted by $\left\{\mathrm{d} x^{i}\right\}$. Thus,

$$
\mathrm{d} x^{i}\left(\partial_{j}\right)=\delta_{j}^{i} .
$$

For each $x \in \mathscr{X}, W_{x}:=\pi^{-1}(x)$ is a vector space that is isomorphic to some fixed $m$-dimensional vector space $\boldsymbol{W}$, although no natural or particular isomorphism is assumed. In particular, for a pair of points $x, y \in \mathscr{X}$, there is no natural isomorphism of $W_{x}$ with $W_{y}$, although both are isomorphic to $\boldsymbol{W}$. The mapping $\pi$ maps all vectors in $W_{x}$ to the point $x$.

Depending on the terminology and context, a vector $w \in W_{x}$ is interpreted either as a virtual velocity/displacement, or as a generalized velocity, or as variation of the field, at the point $x$. It should be mentioned that for the Lagrangian point of view of continuum mechanics on manifolds, the vector bundle $W$ depends on the particular configuration $\kappa$ of the body in space so that $w$ is interpreted as a velocity of the particle $x$ at the point $\kappa(x)$ in space or as a virtual displacement from $\kappa(x)$.

A generalized velocity field is therefore a section, a mapping $w: \mathscr{X} \rightarrow W$ that assigns to each point $x$ a value for its generalized velocity. It follows that $\pi \circ w=$ $\operatorname{Id} \mathscr{x}$, i.e., $\pi(w(x))=x$.

A vector bundle chart, or a coordinate system, will assign to each $w \in W$ a collection of coordinates $\left(x^{i}, w^{\alpha}\right)$, where $x^{i}$ are coordinates for the point $x=\pi(w)$ and $w^{\alpha}, \alpha=1, \ldots, m$, are the components of $w$ relative to some basis $\left\{\boldsymbol{e}_{\alpha}\right\}$ of $W_{x}$. It is assumed that the bases $\left\{\boldsymbol{e}_{\alpha}\right\}$ for the various points $x$ covered by the charts depend on $x$ smoothly. At each point $x$, covered by the charts $\left(x^{i}, w^{\alpha}\right)$ and $\left(x^{i^{\prime}}, w^{\alpha^{\prime}}\right)$, for any $w \in W_{x}$, we must have $w=w^{\alpha} \boldsymbol{e}_{\alpha}=w^{\alpha^{\prime}} \boldsymbol{e}_{\alpha^{\prime}}$ so that there is a matrix $A_{\alpha}^{\alpha^{\prime}}$, depending on $x$, such that $w^{\alpha^{\prime}}=A_{\alpha}^{\alpha^{\prime}} w^{\alpha}$. 
2.2. Why jets. Say $w: \mathscr{X} \rightarrow W$ is a velocity field. The components of $w(x)$ relative to the chart $\left(x^{i}, w^{\alpha}\right)$ are given in terms of $m$ functions $w^{\alpha}\left(x^{i}\right)$. For the chart $\left(x^{i^{\prime}}, w^{\alpha^{\prime}}\right)$, the components are given by the functions $w^{\alpha^{\prime}}\left(x^{i^{\prime}}\right)$ and evidently

$$
w^{\alpha^{\prime}}\left(x^{i^{\prime}}\right)=A_{\alpha}^{\alpha^{\prime}}\left(x^{j}\right) w^{\alpha}\left(x^{j}\right),
$$

where we have indicated explicitly the dependence of the matrix $A_{\alpha}^{\alpha^{\prime}}$ on the point $x$. Differentiating the last identity, using a comma to denote partial derivatives and the summation convention, we obtain

$$
w_{, i^{\prime}}^{\alpha^{\prime}}=A_{\alpha, j}^{\alpha^{\prime}{ }_{, i^{\prime}}} x^{j} w^{\alpha}+A_{\alpha}^{\alpha^{\prime}} w_{, j}^{\alpha} x_{, i^{\prime}}^{j}
$$

This simple relation indicates a fundamental problem. The derivatives $w_{, i^{\prime}}^{\alpha^{\prime}}$ do not depend only on the derivatives $w_{, i}^{\alpha}$; they depend also on the values of $w^{\alpha}$. In other words, while a generalized velocity as a vector field is a well-defined object, the derivative of the generalized velocity is not a well-defined mathematical object (and in particular, as shown in the last equation, the transformation of the partial derivatives may be viewed as an affine transformation). One cannot separate the values of the derivatives from the values of the velocity field in a manner that will be independent of a chart. As an example, we observe that the derivatives may vanish in one coordinate system while they would be different from zero in another. Nevertheless, if we combine the values of the field and the derivatives into a single object, the transformation rules above show that this object - the first jet of the generalized velocity, $j^{1} w$-is well defined. Thus, the first jet of $w$ is represented in the form $\left(x^{i}, w^{\alpha}, w_{, j}^{\alpha}\right)$, or we may write

$$
j^{1} w=w^{\alpha} \boldsymbol{e}_{\alpha}+w_{, i}^{\alpha} d x^{i} \otimes \boldsymbol{e}_{\alpha} .
$$

The collection of 1-jets to the vector bundle $W$ is denoted as $J^{1} W$.

Similarly, we may consider higher-order derivatives of vector fields. In analogy with the case of first derivatives, one realizes that under transformation of coordinates the components of the $k$-th derivatives $w_{, i_{1}^{\prime} \ldots i_{k}}^{\alpha^{\prime}}$ depend on the values of components of all derivatives $w_{, i_{1} \ldots i_{l}}^{\alpha}, 0 \leq l \leq k$, where we identify the zeroth derivative with the value of the function. Thus, the invariant object is the $k$-jet of the velocity field represented under a coordinate system in the form

$$
\begin{aligned}
j^{k} w=w^{\alpha} \boldsymbol{e}_{\alpha} & +w_{, i_{1}}^{\alpha} d x^{i_{1}} \otimes \boldsymbol{e}_{\alpha} \\
& +w_{, i_{1} i_{2}}^{\alpha} d x^{i_{1}} \otimes d x^{i_{2}} \otimes \boldsymbol{e}_{\alpha}+\cdots+w_{, i_{1} \cdots i_{k}}^{\alpha} d x^{i} \otimes \cdots \otimes d x^{i_{k}} \otimes \boldsymbol{e}_{\alpha},
\end{aligned}
$$

or by $\left(x^{i}, w^{\alpha}, w_{, j_{1}}^{\alpha_{1}}, w_{, j_{1} j_{2}}^{\alpha_{2}}, \ldots, w_{, j_{1} \ldots j_{k}}^{\alpha_{k}}\right)$. Formally, a $k$-jet at a point $x \in \mathscr{X}$ is defined as an equivalence class of sections for the equivalence relation by which two sections $w_{1}$ and $w_{2}$ are equivalent if their values at $x$, together with the values of 
the partial derivatives of their local representative under any chart, are equal, respectively. One can show that this definition is independent of the chart chosen (see [Palais 1968; Saunders 1989]). The collection of $k$-jets to $W$ is denoted by $J^{k} W$.

Since higher-order continuum mechanics involves higher-order derivatives of the generalized velocities, we conclude that the terminology of jet bundles provides an appropriate setting for the formulation of such theories.

2.3. Constructions involving jets. Note that each velocity field determines a jet at any given point. Given a chart, the representation of the jet at $x$, determined by the velocity field $w$, is obtained by differentiating the components of $w$ relative to the local coordinates. Any two velocity fields will determine the same $k$-jet at $x$, if their derivatives up to order $k$ are identical.

On the jet bundle, $J^{k} W$ one defines the following mappings. The source map

$$
\pi^{k}: J^{k} W \rightarrow \mathscr{X} \text {, represented by }\left(x^{i}, w^{\alpha}, w_{, j_{1}}^{\alpha_{1}}, w_{, j_{1} j_{2}}^{\alpha_{2}}, \ldots, w_{, j_{1} \ldots j_{k}}^{\alpha_{k}}\right) \mapsto\left(x^{i}\right) \text {, }
$$

assigns to each jet the point in which it is attached. The mapping

$$
\pi_{l}^{k}: J^{k} W \rightarrow J^{l} W, \quad l<k
$$

represented by

$$
\left(x^{i}, w^{\alpha}, w_{, j_{1}}^{\alpha_{1}}, w_{, j_{1} j_{2}}^{\alpha_{2}}, \ldots, w_{, j_{1} \ldots j_{k}}^{\alpha_{k}}\right) \mapsto\left(x^{i}, w^{\alpha}, w_{, j_{1}}^{\alpha_{1}}, w_{, j_{1} j_{2}}^{\alpha_{2}}, \ldots, w_{, j_{1} \ldots j_{l}}^{\alpha_{k}}\right),
$$

assigns to any $k$-jet a jet of a lower order by omitting the derivatives of order higher than $l$. In particular, identifying $J^{0} W$ with $W$, we have

$$
\pi_{0}^{k}: J^{k} W \rightarrow W
$$

which retains only the value of the generalized velocity field itself.

2.4. Symmetric tensors and jets. Henceforth, we will use the notation and ideas introduced in the Appendix to represent, locally, elements of jet bundles. The tensors considered in the Appendix are homogeneous in the sense that they have a definite order, a local representation of a $k$-jet is an element of the symmetric algebra and is represented in general by a collection of symmetric tensors of all orders $l \leq k$. We recall that the representation in (2-7) uses the regular tensor products that are not appropriate base vectors.

The multilinear mappings that represent a jet are not real valued. Rather, they are valued in $\boldsymbol{V}$ - the typical fiber of the vector bundle. We use a local basis $\left\{\boldsymbol{e}_{\alpha}\right\}$ for the vector spaces $W_{x}$ so that a section of $W$ is locally of the form

$$
w=w^{\alpha} \boldsymbol{e}_{\alpha}
$$

where the components $w^{\alpha}$ are real valued functions. This does not affect the symmetry properties considered above. The basic vector space on which the tensors 
are defined at each point is the tangent space of the manifold at that point. Given a chart with coordinates $\left(x^{i}\right)$, the base vectors induced are $\left\{\partial_{i}\right\}$ and they replace the base vectors $\left\{\boldsymbol{e}_{i}\right\}$ used in the Appendix. The various derivatives in $w_{, \boldsymbol{I}}^{\alpha}$ are covariant tensors and are represented using the dual basis $\left\{\mathrm{d} x^{i}\right\}$. The derivatives $w_{, \boldsymbol{I}}^{\alpha}(x),|\boldsymbol{I}|=l$, are elements of

$$
L_{S}^{l}\left(T_{x} \mathscr{X}, W_{x}\right) \simeq \bigodot^{l} T_{x}^{*} \mathscr{X} \otimes W_{x} .
$$

Thus, we may rewrite now (2-7) in the form

$$
j^{k} w=w_{, I}^{\alpha} \overleftarrow{\mathrm{d} x}^{(\boldsymbol{I})} \otimes \boldsymbol{e}_{\alpha}=\overleftarrow{w}_{, \boldsymbol{I}}^{\alpha} \mathrm{d} x^{(\boldsymbol{I})} \otimes \boldsymbol{e}_{\alpha}, \quad 0 \leq|\boldsymbol{I}| \leq k
$$

An element $A \in J^{k} W$ of the jet bundle with $\pi^{k}(A)=x \in \mathscr{X}$ is of the form

$$
A=j_{x}^{k} w:=\left(j^{k} w\right)(x),
$$

for some section $w$ which may be represented locally as

$$
A=w_{, \boldsymbol{I}}^{\alpha}(x) \overleftarrow{\mathrm{d} x}(\boldsymbol{I}) \otimes \boldsymbol{e}_{\alpha}
$$

Noting that the values of the various $w_{, I}^{\alpha}(x)$ are not constrained by compatibility, any element of the jet bundle may be represented in the form

$$
A=A_{\boldsymbol{I}}^{\alpha} \overleftarrow{\mathrm{d} x}^{(\boldsymbol{I})} \otimes \boldsymbol{e}_{\alpha}=\overleftarrow{A}_{\boldsymbol{I}}^{\alpha} \mathrm{d} x^{(\boldsymbol{I})} \otimes \boldsymbol{e}_{\alpha}, \quad 0 \leq|\boldsymbol{I}| \leq k
$$

$A_{\boldsymbol{I}}^{\alpha} \in \bigodot^{|\boldsymbol{I}|} T_{x}^{*} \mathscr{L} \otimes W_{x}$. Given an element of the jet bundle, one can construct a local section, representing it by using the corresponding Taylor polynomial in any chart.

We finally remark that the representation using $\overleftarrow{\mathrm{d} x}^{(\boldsymbol{I})}$ seems preferable because the components of the jet are exactly the derivatives.

2.5. Duality for jets . In view of (A-67), the dual basis of $\left\{\overleftarrow{\mathrm{d} x}^{(\boldsymbol{I})}|0 \leq| \boldsymbol{I} \mid \leq k\right\}$ is $\left\{\partial_{(\boldsymbol{I})}|0 \leq| \boldsymbol{I} \mid \leq k\right\}$. Note that $\partial_{(\boldsymbol{I})}:=\partial_{i_{1}} \odot \cdots \odot \partial_{i_{|\boldsymbol{I}|}}$ is the symmetrized tensor product while $\partial_{\boldsymbol{I}}$ is the differential operator which is symmetric automatically. Real valued linear mappings on the space of jets at a point $x \in \mathscr{X}$ make up the dual space $\left(J_{x}^{k} W\right)^{*}$. Such a linear functional

$$
\varphi: J_{x}^{k} W \rightarrow \mathbb{R}
$$

is locally of the form

$$
\varphi=\varphi_{\alpha}^{\boldsymbol{I}} \partial_{(\boldsymbol{I})} \otimes \boldsymbol{e}^{\alpha},
$$

so that for $\varphi \in\left(J_{x}^{k} W\right)^{*}, A=j^{k} w(x) \in J_{x}^{k} W$,

$$
\varphi(A)=\varphi_{\alpha}^{\boldsymbol{I}} A_{\boldsymbol{I}}^{\alpha}=\varphi_{\alpha}^{\boldsymbol{I}} w_{, \boldsymbol{I}}^{\alpha},
$$

where $0 \leq|\boldsymbol{I}| \leq k$, unless indicated otherwise. 


\section{Variational hyper-stresses}

In accordance with the variational approach to higher-order continuum mechanics, we view variational hyper-stresses as fields that act on the derivatives of the virtual velocities to produce power densities (see [Segev 2017]). Thus, in the current setting, a variational hyper-stress object should act linearly on the $k$-jet of a field $w$ to produce a density on $\mathscr{X}$.

We recall that for integration over an $n$-dimensional manifold, such as $\mathscr{X}$, densities (integrands) are $n$-forms - alternating (completely antisymmetric) tensor fields of order $n$. The space of $r$-alternating tensors over $T_{x} \mathscr{L}$ will be denoted by $\wedge^{r} T_{x}^{*} \mathscr{X}$ and the bundle of alternating tensors is $\bigwedge^{r} T^{*} \mathscr{X}$. A local coordinate system $\left(x^{i}\right)$ induces such an $n$-form

$$
\mathrm{d} x=\mathrm{d} x^{1} \wedge \cdots \wedge \mathrm{d} x^{n},
$$

where a wedge denotes the exterior product — the antisymmetrized tensor product. Note that antisymmetric tensors cannot have repeated indices and so the multiindices representing base vectors and components are strictly increasing rather than nondecreasing. This implies that $\bigwedge^{n} T_{x}^{*} \mathscr{X}$ is one-dimensional, and $\mathrm{d} x$, the $n$-form induced by a local coordinate system, may serve as a basis. Thus, every $n$-form may be written locally as

$$
\theta=\vartheta(x) \mathrm{d} x
$$

for some real valued function $\vartheta$.

In view of these observations, a variational hyper-stress object at $x$ should be a linear mapping

$$
S_{x}: J_{x}^{k} W \rightarrow \bigwedge^{r} T_{x}^{*} \mathscr{X}
$$

so that $S_{x}\left(j^{k} w(x)\right)$ is the power density. Denoting the bundle of linear mappings $J^{k} W \rightarrow \bigwedge^{n} T^{* \mathscr{X}}$ by $L\left(J^{k} W, \bigwedge^{n} T^{*} \mathscr{X}\right)$,

$$
S_{x} \in L\left(J_{x}^{k} W, \bigwedge^{n} T_{x}^{*} \mathscr{X}\right)=L\left(J^{k} W, \bigwedge^{n} T^{*} \mathscr{X}\right)_{x} .
$$

It is also observed that

$$
L\left(J_{x}^{k} W, \bigwedge^{n} T_{x}^{*} \mathscr{X}\right)=\left(J_{x}^{k} W\right)^{*} \otimes \bigwedge^{n} T_{x}^{*} \mathscr{X},
$$

and

$$
L\left(J^{k} W, \bigwedge^{n} T^{*} \mathscr{X}\right)=\left(J^{k} W\right)^{*} \otimes_{\mathscr{X}} \bigwedge^{n} T^{*} \mathscr{X} .
$$

We conclude that a variational hyper-stress field is a section $S$ of $L\left(J^{k} W\right.$, $\left.\bigwedge^{n} T^{*} \mathscr{X}\right)$. In view of the representation of elements of the dual to the jet bundle in Section 2.5, the local representation of $S$ is of the form

$$
S=S_{\alpha}^{\boldsymbol{I}} \partial_{(\boldsymbol{I})} \otimes \boldsymbol{e}^{\alpha} \otimes \mathrm{d} x .
$$


The action of a variational hyper-stress on the jet of a generalized velocity is the density given by

$$
S\left(j^{k} w\right)=S_{\alpha}^{\boldsymbol{I}} w_{, \boldsymbol{I}}^{\alpha} \mathrm{d} x
$$

and the total power is

$$
P=\int_{\mathscr{C}} S \cdot j^{k} w
$$

where $S \cdot j^{k} w$ is the $n$-form $\left(S \cdot j^{k} w\right)(x)=S(x)\left(j^{k} w(x)\right)$.

\section{Traction hyper-stresses and almost symmetric tensors}

The stress object in traditional continuum mechanics plays two roles. On the one hand, from the variational point of view, the stress object acts on the derivative of the velocity field to produce power. The generalization of this object is the variational hyper-stress introduced above. On the other hand, as a result of Cauchy's stress theorem, the stress object determines the traction field on the boundary of the body and its subbodies. While the same mathematical object plays these two roles in the traditional formulation, in the case of a formulation on manifolds, the traction is determined by a different mathematical object - the traction stress (see [Segev 2013]).

4.1. Traction and traction stresses. For the case $k=1$ - first order continuum mechanics - the traction field on the boundary of $\mathscr{X}$, or in general, any of its subbodies (subregions) $\mathscr{R}$, acts linearly on the values of the generalized velocity $w$ to produce a power density over the boundary, the flux of power. Since the boundaries are manifolds of dimensions $n-1$, a power density over the boundary $\partial \mathscr{R}$ is an $(n-1)$-form over $\partial \mathscr{R}$, that is, a section of $\bigwedge^{n-1} T^{*} \partial \mathscr{R}$. Thus, the traction field on the boundary is a section of

$$
L\left(W, \bigwedge^{n-1} T^{*} \partial \mathscr{R}\right),
$$

where, with some abuse of notation, we have omitted the indication that we restrict $W$ to $\partial \mathscr{R}$. It is observed that the fibers of $\bigwedge^{n-1} T^{*} \partial \mathscr{R}$ are one-dimensional.

A traction stress - an object that unlike a traction field is defined over the entire $\mathscr{X}$ - should induce a traction field on the boundary of each subregion using a generalization of Cauchy's formula. A natural candidate for such a mathematical object is suggested by the following observation. While the space of $(n-1)-$ alternating tensors over $\partial \mathscr{R}$ is one-dimensional, the space $\wedge^{n-1} T^{*} \mathscr{L}$ of $(n-1)$ alternating tensors over $\mathscr{X}$ is $n$-dimensional. While an element of $\wedge^{n-1} T^{*} \mathscr{X}$ assigns a value to any collection of $n-1$ vectors, an element of $\wedge^{n-1} T^{*} \partial \mathscr{R}$ assigns values only to vectors tangent to $\partial \mathscr{R}$. In fact, an element of $\bigwedge^{n-1} T^{*} \mathscr{L}$ may be restricted to act on vectors tangent to $\partial \mathscr{R}$ for every subbody $\mathscr{R}$. Thus, for each subbody $\mathscr{R}$, 
we have a restriction mapping

$$
\rho_{\partial \mathscr{R}}: \bigwedge^{n-1} T^{* \mathscr{X}} \rightarrow \bigwedge^{n-1} T^{*} \partial \mathscr{R}
$$

naturally defined by

$$
\rho_{\partial \mathscr{R}}(\omega)\left(v_{1}, \ldots, v_{n}\right)=\omega\left(v_{1}, \ldots, v_{n}\right), \quad v_{r} \in T \partial \mathscr{R} .
$$

Thus, a traction stress is defined to be an element

$$
\sigma_{0} \in L\left(W, \bigwedge^{n-1} T^{*} \mathscr{X}\right) .
$$

Given a traction stress $\sigma_{0}$, at a point $x$, for any subbody $\mathscr{R}$ with $x \in \partial \mathscr{R}$, a traction $\boldsymbol{t}_{0} \in L\left(W, \bigwedge^{n-1} T^{*} \partial \mathscr{R}\right)$ is determined at $x$ by setting

$$
\boldsymbol{t}_{0}=\hat{\rho}_{\partial \mathscr{R}}(\sigma)=\rho_{\partial \mathscr{R}} \circ \sigma, \quad \text { i.e., } \quad \boldsymbol{t}_{0}(w)=\rho_{\partial \mathscr{R}}(\sigma(w)) .
$$

The last equation is the required generalization of Cauchy's formula to the setting of differentiable manifolds. In analogy with the classical Cauchy theorem, it can be shown that if the traction is given on the boundary of every subbody $\mathscr{R}$, with $x \in \partial \mathscr{R}$, then, assuming that certain consistency conditions hold, a unique traction stress is determined at $x$ (see [Segev and Rodnay 1999; Segev 2013] for details).

A traction stress field is a section of the bundle $L\left(W, \bigwedge^{n-1} T^{*} \mathscr{X}\right)$.

4.2. On the local representation of $(n-1)$-forms and traction stresses. Traction stresses are elements of

$$
L\left(W, \bigwedge^{n-1} T^{*} \mathscr{X}\right) \simeq W^{*} \otimes \bigwedge^{n-1} T^{*} \mathscr{X} .
$$

Thus, we make a few comments on the representation of $(n-1)$-alternating tensors, i.e., for a vector space $\boldsymbol{V}$, we consider elements of $\bigwedge^{n-1} \boldsymbol{V}^{*}$.

We first recall that $\wedge^{n} \boldsymbol{V}^{*}$ is one-dimensional and that $\bigwedge^{n-1} \boldsymbol{V}^{*}$ is $n$-dimensional. Let $\lrcorner$ denote the contraction (inner product) whereby for an alternating $r$-tensor $\omega \in \wedge^{r} \boldsymbol{V}^{*}$ and a vector $\left.v_{1} \in \boldsymbol{V}, v_{1}\right\lrcorner \omega$ is the alternating $(r-1)$-tensor such that

$$
\left.v_{1}\right\lrcorner \omega\left(v_{2}, \ldots, v_{r}\right)=\omega\left(v_{1}, \ldots, v_{r}\right) .
$$

In fact, considering the particular case $r=n-1$, one can view the contraction as a mapping

$$
\left.\widehat{\lrcorner}: \boldsymbol{V} \times \bigwedge^{n} \boldsymbol{V}^{*} \rightarrow \bigwedge^{n-1} \boldsymbol{V}^{*}, \quad \widehat{\lrcorner}(v, \theta)=v\right\lrcorner \theta .
$$

We observe that the definition of the contraction mapping implies immediately that the mapping $\widehat{\lrcorner}$ is bilinear. It follows from the universal property of tensor products that there is a linear mapping, which we still denote as $\widehat{\lrcorner}$, such that

$$
\left.\widehat{\lrcorner}: \boldsymbol{V} \otimes \bigwedge^{n} \boldsymbol{V}^{*} \rightarrow \bigwedge^{n-1} \boldsymbol{V}^{*}, \quad \widehat{\lrcorner}(v \otimes \theta)=v\right\lrcorner \theta .
$$


One can verify that this mapping is injective (e.g., [Segev 2013]), and as the dimensions match, it follows that $\widehat{\lrcorner}$ defines a natural isomorphism

$$
\boldsymbol{V} \otimes \bigwedge^{n} \boldsymbol{V}^{*} \simeq \bigwedge^{n-1} \boldsymbol{V}^{*}
$$

Furthermore, for a basis $\left\{\boldsymbol{e}_{i}\right\}$, a natural basis of $\wedge^{n} \boldsymbol{V}^{*}$ is $\boldsymbol{e}^{1} \wedge \cdots \wedge \boldsymbol{e}^{n}$, and so

$$
\left.\left\{\boldsymbol{e}_{i}\right\lrcorner\left(\boldsymbol{e}^{1} \wedge \cdots \wedge \boldsymbol{e}^{n}\right)\right\}, \quad i=1, \ldots, n,
$$

may serve as a natural basis to $\wedge^{n-1} \boldsymbol{V}^{*}$.

Going back to traction stresses, it follows from the foregoing discussion that

$$
L\left(W, \bigwedge^{n-1} T^{*} \mathscr{X}\right) \simeq W^{*} \otimes \bigwedge^{n-1} T^{*} \mathscr{X} \simeq W^{*} \otimes T \mathscr{X} \otimes \bigwedge^{n} T^{*} \mathscr{X} .
$$

For a given coordinate system $\left(x^{i}\right)$, the collection $\left.\left\{\partial_{i}\right\lrcorner \mathrm{d} x\right\}$ may serve as a basis for $\left(\wedge^{n-1} T^{*} \mathscr{X}\right)_{x}$. As a result, any $\omega$ may be represented locally in the form

$$
\left.\omega=\omega^{i} \partial_{i}\right\lrcorner \mathrm{d} x,
$$

where $\mathrm{d} x$ is defined in (3-1). The local representation of a traction stress will be

$$
\left.\sigma=\sigma_{\alpha}^{i} \boldsymbol{e}^{\alpha} \otimes\left(\partial_{i}\right\lrcorner \mathrm{d} x\right)
$$

and

$$
\left.\sigma(w)=\sigma_{\alpha}^{i} w^{\alpha}\left(\partial_{i}\right\lrcorner \mathrm{d} x\right) .
$$

4.3. Hyper-traction and traction hyper-stresses. By analogy with the case $k=1$ described above, where the traction object acts on the $k-1=0$-jet of the generalized velocity, we propose that a hyper-traction on the boundary $\partial \mathscr{R}$ of a subbody $\mathscr{R}$, be defined as an element

$$
\boldsymbol{t} \in L\left(J^{k-1} W, \bigwedge^{n-1} T^{*} \partial \mathscr{R}\right) \simeq\left(J^{k-1} W\right)^{*} \otimes \bigwedge^{n-1} T^{*} \partial \mathscr{R} .
$$

Thus, the total power flux is given by

$$
\int_{\partial \mathscr{R}} \boldsymbol{t} \cdot j^{k-1} w
$$

A traction hyper-stress field is defined in analogy with the definition of a traction stress, in the sense that it acts on a lower order jet to give an $(n-1)$-form which can be integrated on the boundaries of subbodies. Thus, a traction hyper-stress is defined to be an element

$$
\sigma_{0} \in L\left(J^{k-1} W, \bigwedge^{n-1} T^{*} \mathscr{X}\right) \simeq\left(J^{k-1} W\right)^{*} \otimes T \mathscr{X} \otimes \bigwedge^{n} T^{*} \mathscr{X} .
$$

It follows from the foregoing analysis that a traction hyper-stress is represented locally in the form

$$
\left.\sigma_{0}=\sigma_{\alpha}^{J j} \partial_{(\boldsymbol{J})} \otimes \boldsymbol{e}^{\alpha} \otimes\left(\partial_{i}\right\lrcorner \mathrm{d} x\right), \quad 0 \leq|\boldsymbol{J}| \leq k-1 .
$$


A traction hyper-stress field is a section of $L\left(J^{k-1} W, \bigwedge^{n-1} T^{*} \mathscr{X}\right)$ and the action of a hyper-stress field $\sigma$ on the $(k-1)$-jet of a generalized velocity $w$ is given by

$$
\left.\sigma \cdot j^{k-1} w=\sigma_{\alpha}^{J j} w_{, J}^{\alpha} \partial_{i}\right\lrcorner \mathrm{d} x .
$$

These natural extensions imply that the Cauchy formula (4-5) remains applicable as it simply represents the restriction of forms. Thus, given a traction hyper-stress field $\sigma$ and a generalized velocity field $w$, the total flux of power through the boundary $\partial \mathscr{R}$ is

$$
\int_{\partial \mathscr{R}} \boldsymbol{t} \cdot j^{k-1} w=\int_{\partial \mathscr{R}} \hat{\rho}_{\partial \mathscr{R}}(\sigma) \cdot j^{k-1} w .
$$

It is emphasized that the array $\sigma_{\alpha}^{J j}$ representing a traction hyper-stress is symmetric with respect to permutations of the multi-index $\boldsymbol{J}$ and for this reason it appears in conjunction with the symmetrized basis $\partial_{(J)}$. In particular, no symmetry is expected for permutations that "mix" the indices $\boldsymbol{J}$ and $j$. Thus, for a fixed value $l=|\boldsymbol{J}|$, we refer to the tensor $\sigma_{\alpha}^{J j}$ as the almost symmetric tensor.

4.4. Almost symmetric tensors. In order to simplify the notation we will consider henceforth only real valued almost symmetric tensors. That is, for some given vector space $\boldsymbol{V}$ we consider elements of $\left(\bigodot^{l-1} \boldsymbol{V}\right) \otimes \boldsymbol{V}$ rather than elements of $\left(\bigodot^{l-1} \boldsymbol{V}\right) \otimes \boldsymbol{V}^{*} \otimes \boldsymbol{V} \otimes \bigwedge^{n} \boldsymbol{V}^{*}$

Let $\left\{\boldsymbol{e}_{i}\right\}$ be a basis in $\boldsymbol{V}$. Then, we may use either $\left\{\boldsymbol{e}_{(\boldsymbol{J})}\right\}, 0 \leq|\boldsymbol{J}| \leq l-1$, or the basis $\left\{\overleftarrow{\boldsymbol{e}}_{(\boldsymbol{J})}\right\}$ for $\bigodot^{l-1} \boldsymbol{V}$ in analogy with (A-56). A real valued almost symmetric tensor $T$ can be represented in the form

$$
T=T^{I} \boldsymbol{e}_{I}=T^{\boldsymbol{J} j} \overleftarrow{\boldsymbol{e}}_{(\boldsymbol{J})} \otimes \boldsymbol{e}_{j}=\overleftarrow{T}^{\boldsymbol{J} j} \boldsymbol{e}_{(\boldsymbol{J})} \otimes \boldsymbol{e}_{j}
$$

where $0 \leq|\boldsymbol{J}| \leq l-1,0 \leq|I| \leq l$, and

$$
\overleftarrow{\boldsymbol{e}}_{(\boldsymbol{J})}=\frac{(l-1) !}{\boldsymbol{J} !} \boldsymbol{e}_{(\boldsymbol{J})}, \quad \overleftarrow{T}^{\boldsymbol{J}}=\frac{(l-1) !}{\boldsymbol{J} !} T^{J}
$$

For the dual space we have

$$
\left[\left(\bigodot^{l-1} \boldsymbol{V}\right) \otimes \boldsymbol{V}\right]^{*} \simeq\left(\bigodot^{l-1} \boldsymbol{V}^{*}\right) \otimes \boldsymbol{V}^{*}
$$

so that its elements may be referred to as almost symmetric cotensors. For the basis $\left\{\overleftarrow{\boldsymbol{e}}_{(\boldsymbol{J})} \otimes \boldsymbol{e}_{j}\right\}$, the dual basis will be $\left\{\boldsymbol{e}^{(\boldsymbol{J})} \otimes \boldsymbol{e}^{j}\right\}$. An element $\varphi$ of $\left[\left(\bigodot^{l-1} \boldsymbol{V}\right) \otimes \boldsymbol{V}\right]^{*}$ is represented in the form

$$
\varphi=\varphi_{\boldsymbol{J} j} \boldsymbol{e}^{(\boldsymbol{J})} \otimes \boldsymbol{e}^{j}
$$

with

$$
\varphi(T)=\varphi_{\boldsymbol{J} j} T^{\boldsymbol{J} j} .
$$




\section{Conclusion}

We have reviewed above the language needed for the formulation of higher-order continuum mechanics on differentiable manifolds. In particular, we have proposed the mathematical object that we believe should play the role of traction hyper-stress. While for the case $k=1$, the traction stress has been defined in [Segev 2002; 2013], no natural analogous definition has been presented in [Segev 2017]. In fact, in [Segev 2017] some of the difficulties have been indicated and subsequently avoided by using iterated jet bundles (the jet bundle of the jet bundle) and the corresponding dual objects rather than analyzing directly higher jet bundles and hyper-stresses.

Nevertheless, no relation between variational hyper-stresses and the proposed traction hyper-stresses has been given above. We hope to study this relation in a forthcoming work.

\section{Appendix: Notes on symmetric tensors}

As the local representation of jets involves iterated partial differentiation, symmetric tensors are of major importance. In these notes we review the basic properties of symmetric tensors and the relevant notation as we use in the main text.

A.1. Multi-index notation. Multi-index notation is very effective when high-order tensors are involved, as is the situation here. A multi-index $I$ of length $k$ is a $k$-tuple of positive integers, e.g., $I=i_{1} \ldots i_{k}$. Multi-indices will be denoted by upper-case roman letters and the associated indices will be denoted by the corresponding lower case letters as in the example above. For example, we may write the components $T_{i j k}$ of a third order tensor $T$ as $T_{I}=T_{i_{1} i_{2} i_{3}}$. The length of a multi-index $I=i_{1} \ldots i_{k}$ is denoted as the absolute value of the multi-index, i.e., $|I|=k$. We will use the summation convention for multi-indices so the contraction of two tensors may be written as $T^{I} S_{I}$. When a multi-index appears more than twice in a term, or twice but not diagonally, it is implied that the summation convention for that multi-index is not in effect.

Multi-indices may be concatenated naturally so that for two multi-indices $I$ and $J$, the concatenated multi-index is $I J=i_{1} \ldots i_{|I|} j_{1} \ldots j_{|J|}$ whose length is $|I J|=|I|+|J|$. Thus, for two tensors $S_{I}$ and $T_{J}$, one may write $(S \otimes T)_{I J}=S_{I} T_{J}$.

For two multi-indices $I, J$, with $|I|=|J|=l$, one extends the definition of the Kronecker $\delta$ by

$$
\delta_{J}^{I}:=\delta_{j_{1}}^{i_{1}} \cdots \delta_{j_{l}}^{i_{l}}
$$

A.2. Symmetric tensors and permutations. Because of the commutativity of partial derivatives that we encounter frequently here, tensors that are completely symmetric are of particular interest. A tensor $T$ is completely symmetric if for any exchange of two indices $i_{r}$ and $i_{s}, T_{i_{1} \cdots i_{r} \cdots i_{s} \cdots i_{k}}=T_{i_{1} \cdots i_{s} \cdots i_{r} \cdots i_{k}}$. 
Symmetry can also be defined in terms of permutations. A permutation of the finite ordered set $(1, \ldots, l)$ is a bijection

$$
p:(1, \ldots, l) \rightarrow(1, \ldots, l) .
$$

The collection of all such permutations will be denoted by $\mathscr{P}_{l}$. From elementary combinatorics it follows that there are $l$ ! permutations in $\mathscr{P}_{l}$. For a multi-index $I$ and a permutation $p$, we set

$$
p(I):=I \circ p=i_{p(1)} \cdots i_{p(l)} .
$$

Note that $i_{p(r)}$ identifies the index that arrived under the permutation at the $r$-th position, while $i_{p^{-1}(s)}$ is the position of $i_{s}$ after the permutation $p$. Note also that we make some abuse of notation by using the same symbol for the permutation and its action on multi-indices. It immediately follows that for two permutations $p_{1}, p_{2} \in \mathscr{P}_{l}$,

$$
p_{2} \circ p_{1}(I)=I \circ p_{1} \circ p_{2} .
$$

Thus, using the language of permutations, a tensor is symmetric if for every permutation $p \in \mathscr{P}_{l}$,

$$
T_{p(I)}=T_{I} .
$$

Remark 1. We have defined symmetry above in terms of the components of the array representing a tensor. Viewed as a multilinear mapping, a (covariant) tensor $T$ is symmetric if

$$
T\left(v_{1}, \ldots, v_{l}\right)=T\left(v_{p(1)}, \ldots, v_{p(l)}\right)
$$

for any permutation $p$. In particular, for a symmetric tensor

$$
T_{i_{1} \cdots i_{1}}=T\left(\boldsymbol{e}_{i_{1}}, \ldots, \boldsymbol{e}_{i_{l}}\right)=T\left(\boldsymbol{e}_{p\left(i_{1}\right)}, \ldots, \boldsymbol{e}_{p\left(i_{l}\right)}\right)=T_{p\left(i_{1}\right) \cdots p\left(i_{l}\right)}
$$

(see also [Greub 1978]).

We will use the notation $\otimes^{l} \boldsymbol{V}$ for the space of contravariant $l$-tensors and $\bigodot^{l} \boldsymbol{V}$ for the subspace of symmetric tensors. We will also identify a tensor $T \in \bigotimes^{l} \boldsymbol{V}$ with the (possibly symmetric) multilinear mapping $\boldsymbol{V}^{*} \times \cdots \times \boldsymbol{V}^{*} \rightarrow \mathbb{R}$ in the space of (respectively, symmetric) multilinear mappings $L^{l}\left(\boldsymbol{V}^{*}, \mathbb{R}\right)$ (respectively, $\left.L_{S}^{l}\left(\boldsymbol{V}^{*}, \mathbb{R}\right)\right)$. Thus, we make the identifications

$$
\bigotimes^{l} \boldsymbol{V} \simeq L^{l}\left(\boldsymbol{V}^{*}, \mathbb{R}\right), \quad \bigodot^{l} \boldsymbol{V} \simeq L_{S}^{l}\left(\boldsymbol{V}^{*}, \mathbb{R}\right) .
$$

The inclusion of the symmetric tensors will be denoted as

$$
\iota_{S}: \bigodot^{l} \boldsymbol{V} \simeq L_{S}^{l}\left(\boldsymbol{V}^{*}, \mathbb{R}\right) \rightarrow \bigotimes^{l} \boldsymbol{V} \simeq L^{l}\left(\boldsymbol{V}^{*}, \mathbb{R}\right) .
$$

An analogous notation and terminology will be used for covariant tensors. 
Remark 2. The Levi-Civita symbol satisfies ${ }^{1}$

$$
\varepsilon_{J}^{I}=\varepsilon_{j_{1} \ldots j_{m}}^{i_{1} \ldots i_{l}}= \begin{cases}(-1)^{p} & \text { if there is a permutation } p \text { with } J=p(I), \\ 0 & \text { otherwise. }\end{cases}
$$

Thus, we set

$$
|\varepsilon|_{J}^{I}:=\left|\varepsilon_{j_{1} \ldots j_{m}}^{i_{1} \ldots i_{l}}\right|= \begin{cases}1 & \text { if there is a permutation } p \text { with } J=p(I), \\ 0 & \text { otherwise. }\end{cases}
$$

In particular,

$$
|\varepsilon|_{J}^{p(I)}=|\varepsilon|_{J}^{I}
$$

A.3. Cardinality sequence of a multi-index. A multi-index $I$ induces another sequence $\left(I_{1}, \ldots, I_{n}\right), n=\operatorname{dim} \boldsymbol{V}$, the cardinality sequence, in which $I_{r}$ indicates the number of times $r$ is included in the multi-index. Evidently, $|I|=\sum_{r=1}^{n} I_{r}$ and the sequence $\left(I_{1}, \ldots, I_{n}\right)$ is invariant under permutations of the multi-index.

A collection $\left(I_{1}, \ldots, I_{n}\right)$ induces a unique nondecreasing multi-index, i.e., the multi-index

$$
1 \cdots 12 \cdots 2 \cdots \cdots n \cdots n
$$

where the number $r$ appears $I_{r}$ times. Thus, if $\left(I_{1}, \ldots, I_{n}\right)$ is the cardinality sequence of $I$, we obtain a nondecreasing permutation of $I$.

For a multi-index $I$ it is useful to write

$$
I !=I_{1} ! \cdots I_{n} !
$$

It is observed that for a concatenated index $I J$, one has $(I J)_{r}=I_{r}+J_{r}, r=1, \ldots, n$. The index $i=1, \ldots, n$, is a simple multi-index $I=i$. Obviously $|I|=1$ and

$$
I_{r}= \begin{cases}0 & \text { for } r \neq i \\ 1 & \text { for } r=i\end{cases}
$$

Thus, for the concatenated multi-index $J i$, one has $(J i)_{r}=J_{r}+\delta_{r i}$, where $\delta$ is the Kronecker symbol.

For tensors that are symmetric with respect to a multi-index, a particular component is indicated uniquely by a sequence in the form $\left(I_{1}, \ldots, I_{n}\right)$ and by restricting the sequences $\left(i_{1}, \ldots, i_{|\boldsymbol{I}|}\right)$ to be nondecreasing. Consequently, we will use multiindices indicated by bold characters to be nondecreasing only and we will also write $\boldsymbol{I}=\left(I_{1}, \ldots, I_{n}\right)$. In addition, the fact that a multi-index is nondecreasing will be indicated by angle brackets, e.g., $T_{\langle J\rangle}$ or $T_{\langle\boldsymbol{I} \boldsymbol{J}\rangle}$, independently of the symmetry property of a tensor.

${ }^{1}$ This is a somewhat generalized presentation of the Levi-Civita symbol, for which see [LeviCivita 1927, p. 158]. 
A.4. Derivatives. Nondecreasing multi-indices are primarily used for notation involving partial derivatives. We will use the notation

$$
(\cdot)_{, \boldsymbol{I}}=\partial_{\boldsymbol{I}}(\cdot)=\frac{\partial^{|\boldsymbol{I}|}(\cdot)}{\partial x^{\boldsymbol{I}}}=\frac{\partial^{|\boldsymbol{I}|}(\cdot)}{\partial x^{i_{1}} \cdots \partial x^{i_{|I|}}}=\frac{\partial^{|\boldsymbol{I}|}(\cdot)}{\left(\partial x^{1}\right)^{I_{1}} \cdots\left(\partial x^{n}\right)^{I_{n}}} .
$$

Nondecreasing multi-indices may be added naturally by setting

$$
\boldsymbol{I}+\boldsymbol{J}=\left(I_{1}+J_{1}, \ldots, I_{n}+J_{n}\right),
$$

which determines a unique nondecreasing multi-index such that $|\boldsymbol{I}+\boldsymbol{J}|=|\boldsymbol{I}|+|\boldsymbol{J}|$. In particular,

$$
\left((\cdot)_{, \boldsymbol{I}}\right)_{, \boldsymbol{J}}=(\cdot)_{,\langle\boldsymbol{I} \boldsymbol{J}\rangle}=(\cdot),\langle\boldsymbol{I}+\boldsymbol{J}\rangle \cdot
$$

Nondecreasing multi-indices can also be partially ordered so that

$$
\boldsymbol{J} \leq \boldsymbol{I} \quad \Longleftrightarrow \quad J_{r} \leq I_{r} \text { for } r=1, \ldots, n .
$$

In the case $\boldsymbol{J} \leq \boldsymbol{I}$, one can use the subtraction $\boldsymbol{I}-\boldsymbol{J}$.

As hinted in the notation for partial derivatives, for $x \in \mathbb{R}^{n}$, one defines for a nondecreasing multi-index $\boldsymbol{I}$,

$$
x^{I}=\left(x^{1}\right)^{I_{1}} \cdots\left(x^{n}\right)^{I_{n}} .
$$

The summation convention will be applied for bold faced multi-indices, accordingly, only to the nondecreasing sequences. For example, a polynomial $\mathbb{R}^{n} \rightarrow \mathbb{R}$ of order $l$ may be written as

$$
u=a_{I} x^{I}, \quad 0 \leq|\boldsymbol{I}| \leq l .
$$

Suspending the summation convention, its derivatives are

$$
u, \boldsymbol{J}=\sum_{0 \leq|\boldsymbol{I}| \leq l} \frac{\boldsymbol{I} !}{(\boldsymbol{I}-\boldsymbol{J}) !} x^{\boldsymbol{I}-\boldsymbol{J}} .
$$

Although this relation is used mainly for the case where $J_{r} \leq I_{r}$, for all $r=1, \ldots, n$, it may be extended to all other cases by adopting the convention that

$$
\frac{1}{i !}=0 \quad \text { for } i<0 \text {. }
$$

The notation introduced above allows one to write the $l$-th order Taylor expansion of a function $f: \mathbb{R}^{n} \rightarrow \mathbb{R}$ in the form

$$
\sum_{0 \leq \boldsymbol{I} \leq l} \frac{1}{\boldsymbol{I} !} f_{, \boldsymbol{I}}(x) h^{\boldsymbol{I}} .
$$

To use the summation convention, one first sets $g_{\boldsymbol{I}}:=f_{, \boldsymbol{I}} / \boldsymbol{I}$ ! (no sum), and so the polynomial is written as

$$
g_{I} h^{I}, \quad 0 \leq|\boldsymbol{I}| \leq l .
$$


A.5. More on permutations. One observes that, for some given $I,|I|=l$, the sum

$$
\sum_{p \in \mathscr{P}_{l}} T_{p(I)}
$$

contains $l !=|I|$ ! terms, the number of all permutations. These include $I$ ! permutations (see below) that leave $I$ invariant. In the particular case where $T$ is symmetric,

$$
\sum_{p \in \mathscr{P}_{l}} T_{p(I)}=|I| ! T_{I}, \quad \text { no sum on } \boldsymbol{I} .
$$

On the other hand, in the expression

$$
|\varepsilon|_{I}^{J} T_{J}=\sum_{J, J=p(I)} T_{J}
$$

the sum applies only to possible values of the multi-index $J$, irrespective of the number of permutations of $I$ that give it. Assume that $J$ is a permutation of $I$ so that $|\varepsilon|_{I}^{J}=1$. As both $I$ and $J$ contain $I_{r}$ occurrences of the index $r$, permutations of which leave a multi-index invariant, there are $J !=J_{1} ! \cdots J_{l} !=I$ ! such permutations for each $J$. Since there are $I$ ! permutations that give any one particular multi-index $J$ if $|\varepsilon|_{I}^{J} \neq 0$, it follows that for any fixed $J$,

$$
\sum_{\substack{p(I)=J, p \in \mathscr{P}_{l}}} T_{p(I)}=I ! T_{J}=I !|\varepsilon|_{I}^{J} T_{J}, \quad \text { no sum on } I, J,
$$

and so

$$
\sum_{p \in \mathscr{P}_{l}} T_{p(I)}=\sum_{J}\left(\sum_{\substack{p(I)=J, p \in \mathscr{P}_{l}}} T_{p(I)}\right)=I !|\varepsilon|_{I}^{J} T_{J}, \quad \text { no sum on } I .
$$

We conclude that the number of nontrivial terms in the sum $|\varepsilon|_{I}^{J} T_{J}$ is

$$
\sum_{J}|\varepsilon|_{I}^{J}=\frac{|I| !}{I !}=\frac{l !}{I !} .
$$

In the particular case where $T$ is symmetric, $\sum_{p \in \mathscr{P}_{l}} T_{p(I)}=|I| ! T_{I}$, so that (A-30) implies immediately that

$$
\sum_{J, J=p(I)} T_{J}=|\varepsilon|_{I}^{J} T_{J}=\frac{|I| !}{I !} T_{I}
$$

For a given pair of multi-indices, $I, J$, and a variable permutation $p$,

$$
\delta_{p(J)}^{I}=|\varepsilon|_{J}^{I} .
$$


As a result

$$
\sum_{p \in \mathscr{P}_{l}} \delta_{p(J)}^{I}=I !|\varepsilon|_{J}^{I}, \quad \text { no sum on } I .
$$

Remark 3. For each nondecreasing multi-index $\boldsymbol{I},|\boldsymbol{I}|=l$, there are $|\boldsymbol{I}| ! / \boldsymbol{I}$ ! distinct indices $J$. Thus, the total number of distinct multi-indices is

$$
\sum_{I} \frac{\left(I_{1}+\cdots+I_{n}\right) !}{I_{1} ! \cdots I_{n} !}=n^{l},
$$

in accordance with the multinomial formula.

A.6. Symmetrization of arrays and tensors. Any $l$-tensor $T$, having the components $T_{I}$, induces a unique symmetric array, the components of which are denoted as $T_{(I)}$, by

$$
T_{(I)}=\sum_{p \in \mathscr{P}_{l}} \frac{1}{l !} T_{p(I)}=\frac{I !}{l !}|\varepsilon|_{I}^{J} T_{J}, \quad \text { no sum on } \boldsymbol{I} .
$$

We first show that $T_{(I)}$ is indeed symmetric. One has,

$$
T_{(q(I))}=\sum_{p \in \mathscr{P}_{l}} \frac{1}{l !} T_{p(q(I))}=\sum_{p \in \mathscr{P}_{l}} \frac{1}{l !} T_{p(I)}=T_{(I)},
$$

where in the second equality we used the fact that in the first equality we add up the terms over all permutations anyhow. One also observes that symmetrization is a projection in the sense that the symmetrization of a symmetric tensor yields the tensor itself. That is, if $T_{I}$ is symmetric,

$$
T_{(I)}=\sum_{p \in \mathscr{P}_{l}} \frac{1}{l !} T_{p(I)}=\sum_{p \in \mathscr{P}_{l}} \frac{1}{l !} T_{I}=T_{I} .
$$

The symmetrization of a multilinear mapping $T$ - a covariant tensor - is defined as the linear mapping

such that

$$
\mathscr{S}: \bigotimes^{l} \boldsymbol{V}^{*} \rightarrow \bigodot^{l} \boldsymbol{V}^{*}
$$

$$
(\mathscr{S}(T))\left(v_{1}, \ldots, v_{l}\right)=\frac{1}{l !} \sum_{p \in \mathscr{P}_{l}} T\left(v_{p(1)}, \ldots, v_{p(l)}\right) .
$$

In particular,

$$
\begin{aligned}
\mathscr{S}\left(\boldsymbol{e}^{I}\right)\left(v_{1}, \ldots, v_{l}\right) & :=\mathscr{Y}\left(\boldsymbol{e}^{i_{1}} \otimes \cdots \otimes \boldsymbol{e}^{i_{l}}\right)\left(v_{1}, \ldots, v_{l}\right) \\
& =\frac{1}{l !} \sum_{p \in \mathscr{P}_{l}}\left(\boldsymbol{e}^{i_{1}} \otimes \cdots \otimes \boldsymbol{e}^{i_{l}}\right)\left(v_{p(1)}, \ldots, v_{p(l)}\right) \\
& =\frac{1}{l !} \sum_{p \in \mathscr{P}_{l}}\left(\boldsymbol{e}^{p\left(i_{1}\right)} \otimes \cdots \otimes \boldsymbol{e}^{p\left(i_{l}\right)}\right)\left(v_{1}, \ldots, v_{l}\right),
\end{aligned}
$$


and it follows that (see [Greub 1978, p. 219])

$$
\mathscr{S}\left(\boldsymbol{e}^{I}\right)=\frac{1}{l !} \sum_{p \in \mathscr{P}_{l}}\left(\boldsymbol{e}^{p\left(i_{1}\right)} \otimes \cdots \otimes \boldsymbol{e}^{p\left(i_{l}\right)}\right)=: \boldsymbol{e}^{i_{1}} \odot \cdots \odot \boldsymbol{e}^{i_{l}}=: \bigodot^{I} \boldsymbol{e}^{I}=: \boldsymbol{e}^{(I)} .
$$

From this definition it follows immediately that

$$
\boldsymbol{e}_{(p(J))}=\boldsymbol{e}_{(J)} \text { for all } \quad p \in \mathscr{P} .
$$

Hence, $\boldsymbol{e}_{(J)}$ as well as all $\boldsymbol{e}_{(p(J))}$ are represented by the nondecreasing multi-index $\boldsymbol{J}=\langle\boldsymbol{J}\rangle=\left(J_{1}, \ldots, J_{n}\right)$.

Note that for a permutation $p \in \mathscr{P}_{l}$ and a multilinear mapping $T$, one may write $p T$ for the multilinear mapping defined by

$$
(p T)\left(v_{1}, \ldots, v_{l}\right):=T\left(v_{p(1)}, \ldots, v_{p(l)}\right) .
$$

Thus,

$$
\mathscr{S}(T)=\frac{1}{l !} \sum_{p \in \mathscr{P}_{l}} p T, \quad \boldsymbol{e}^{(I)}=\frac{1}{l !} \sum_{p \in \mathscr{P}_{l}} p \boldsymbol{e}^{I}=\frac{1}{l !} \sum_{p \in \mathscr{P}_{l}} \boldsymbol{e}^{p(I)} .
$$

The inclusion of the subspace of symmetric tensors will be denoted by

$$
\iota_{S}: \bigodot^{l} \boldsymbol{V}^{*} \rightarrow \bigotimes^{l} \boldsymbol{V}^{*} .
$$

Since the symmetrization of a symmetric tensor gives the original tensor, the symmetrization mapping $\mathscr{S}$ is a left inverse of the inclusion, i.e., $\mathscr{S} \circ \iota_{S}=\mathrm{Id}$.

It is readily verified that the array $\mathscr{S}(T)_{I}$ of a symmetrized multilinear mapping is the symmetrized array $T_{(I)}$.

A.7. Bases and dimension. We consider a vector space $\boldsymbol{V}$ with some basis $\left\{\boldsymbol{e}_{i}\right\}$, $i=1, \ldots, n$. Let $T$ be a (say contravariant) tensor $T$ of degree $l$ represented in the form

$$
T=T^{i_{1} \cdots i_{l}} \boldsymbol{e}_{i_{1}} \otimes \cdots \otimes \boldsymbol{e}_{i_{l}} .
$$

Using multi-index notation,

$$
T=T^{I} \boldsymbol{e}_{l}, \quad|I|=l,
$$

where

$$
\boldsymbol{e}_{I}:=\boldsymbol{e}_{i_{1}} \otimes \cdots \otimes \boldsymbol{e}_{i_{l}} .
$$

In particular, the dimension of the space is $n$.

The array of a symmetric tensor is uniquely determined by its components $T^{\boldsymbol{I}}$ for nondecreasing multi-indices only. Thus, the dimension of the space of symmetric $l$-tensors is obviously smaller. Since a nondecreasing $\boldsymbol{I}$ is uniquely determined by $I_{1}, \ldots, I_{n}$, the dimension may be determined accordingly. 
It is easy to realize that the number of independent component in a symmetric $l$-tensor is $C(n+l-1, l)=(n+l-1) ! /(n-1) ! l !$. One considers a string of $l$ nondecreasing indices, $I_{1}$ occurrences of $1, I_{2}$ occurrences of 2 , etc., where the end of each such group (except for the last one) is indicated by a divider. Thus, the number of distinct nondecreasing multi-indices is the number of different ways one can place the $n-1$ (identical) dividers in the string containing $l+n-1$ elements (both indices and dividers). It follows that the dimension of the space of symmetric $l$-tensors is $C(n+l-1, l)$.

Since a symmetric tensor is represented by a symmetric array,

$$
\begin{aligned}
T & =T^{I} \boldsymbol{e}_{I}=T^{(I)} \boldsymbol{e}_{I}=\frac{1}{l !} \sum_{p \in \mathscr{P}_{l}} T^{p(I)} \boldsymbol{e}_{I} \\
& =\frac{1}{l !} \sum_{p \in \mathscr{P}_{l}} T^{J} \boldsymbol{e}_{p^{-1}(J)}=\frac{1}{l !} T^{J} \sum_{q \in \mathscr{P}_{l}} \boldsymbol{e}_{q(J)}=T^{J} \boldsymbol{e}_{(J)},
\end{aligned}
$$

where in the second line we used the fact that the order of the sum of the multi-index and the sum over the group of permutations may be reversed. Here, in accordance with (A-42),

$$
\boldsymbol{e}_{(J)}=\bigodot^{J} \boldsymbol{e}_{J}:=\frac{1}{l !} \sum_{q \in \mathscr{P}_{l}} \boldsymbol{e}_{q(J)},
$$

or explicitly

$$
\boldsymbol{e}_{(J)}=\boldsymbol{e}_{j_{1}} \odot \cdots \odot \boldsymbol{e}_{j_{l}}:=\frac{1}{l !} \sum_{q \in \mathscr{P}_{l}} \boldsymbol{e}_{j_{q(1)}} \otimes \cdots \otimes \boldsymbol{e}_{j_{q(l)}},
$$

denotes the symmetric tensor product (see [Greub 1978, p. 219]).

Furthermore,

$$
\begin{aligned}
T & =T^{J} \boldsymbol{e}_{(J)} \\
& =\sum_{\boldsymbol{I}} \sum_{\substack{J=p(\boldsymbol{I}) \\
p \in \mathscr{P}_{l}}} T^{J} \boldsymbol{e}_{(J)} \quad(\text { no sum }) \\
& =\sum_{\boldsymbol{I}} \sum_{\substack{J=p(\boldsymbol{I}) \\
p \in \mathscr{P}_{l}}} T^{J} \boldsymbol{e}_{(\boldsymbol{I})} \quad\left(\text { as } \boldsymbol{e}_{(p(\boldsymbol{I}))}=\boldsymbol{e}_{(\boldsymbol{I})}\right) \\
& =\sum_{\boldsymbol{I}}\left(\sum_{\substack{J=p(\boldsymbol{I}) \\
p \in \mathscr{P}_{l}}} T^{J}\right) \boldsymbol{e}_{(\boldsymbol{I})} \\
& \left.=\sum_{\boldsymbol{I}} \frac{|\boldsymbol{I}| !}{\boldsymbol{I} !} T^{\boldsymbol{I} \boldsymbol{e}_{(\boldsymbol{I})}} \quad \text { (by }(\mathrm{A}-32)\right) .
\end{aligned}
$$


The last expression suggests that we make the definitions

$$
\begin{aligned}
\overleftarrow{\boldsymbol{e}}_{(\boldsymbol{I})}:=\frac{|\boldsymbol{I}| !}{\boldsymbol{I} !} \boldsymbol{e}_{(I)}, \quad \overleftarrow{T}^{\boldsymbol{I}}:=\frac{|\boldsymbol{I}| !}{\boldsymbol{I} !} T^{\boldsymbol{I}}, \\
\overrightarrow{\boldsymbol{e}}_{(J)}:=\frac{J !}{|J| !} \boldsymbol{e}_{(J)}, \quad \vec{T}^{J}:=\frac{J !}{|J| !} T^{J}
\end{aligned}
$$

and it is noted that the fractions $J ! /|J|$ ! are identical for all $J=p(\boldsymbol{I}), p \in \mathscr{P}$. Utilizing the summation convention again, we may write

$$
\begin{gathered}
T=T^{J} \boldsymbol{e}_{(J)}=T^{I} \overleftarrow{\boldsymbol{e}}_{(\boldsymbol{I})}=\overleftarrow{T}^{\boldsymbol{I}} \boldsymbol{e}_{(\boldsymbol{I})} \\
T^{J} \overrightarrow{\boldsymbol{e}}_{(J)}=\vec{T}^{J} \boldsymbol{e}_{(J)}=T^{\boldsymbol{I}} \boldsymbol{e}_{(\boldsymbol{I})}
\end{gathered}
$$

Evidently, both $\left\{\boldsymbol{e}_{(I)}\right\}$, and $\left\{\overleftarrow{\boldsymbol{e}}_{(\boldsymbol{I})}\right\}$ are collections of linearly independent tensors and may serve as bases for the space of symmetric tensors (see [Greub 1978; Comon et al. 2008, p. 219]). The components of the tensor relative to these bases change accordingly. The representation of a symmetric tensor in (A-47) is in terms of regular tensor products and is inadequate because these tensor products are not elements of the space of symmetric tensors, in general, and because it uses more elements than the dimension of the space. The appropriate representation of symmetric tensors in terms of base elements is given by (A-56).

Example 4. We consider now the inclusion

$$
\iota_{S}: \bigodot^{l} \boldsymbol{V} \rightarrow \bigotimes^{l} \boldsymbol{V}
$$

The matrix of the inclusion relative to the bases $\boldsymbol{e}_{J}$ in $\bigotimes^{l} \boldsymbol{V}$ and $\overleftarrow{\boldsymbol{e}}_{(\boldsymbol{I})}$ in $\bigodot^{l} \boldsymbol{V}$ satisfies

$$
\begin{array}{rlr}
\left(\iota_{S}\right)_{\boldsymbol{I}}^{J} \boldsymbol{e}_{J} & =\iota_{S}\left(\overleftarrow{\boldsymbol{e}}_{(\boldsymbol{I})}\right)=\frac{|\boldsymbol{I}| !}{\boldsymbol{I} !} \iota_{S}\left(\boldsymbol{e}_{(\boldsymbol{I})}\right)=\frac{|\boldsymbol{I}| !}{\boldsymbol{I} !} \boldsymbol{e}_{(\boldsymbol{I})} \\
& =\frac{1}{\boldsymbol{I} !} \sum_{p \in \mathscr{P}_{l}} \boldsymbol{e}_{p(\boldsymbol{I})} \quad(\text { no sum on } \boldsymbol{I}) \\
& =\frac{1}{\boldsymbol{I} !} \sum_{p \in \mathscr{P}_{l}} \delta_{p(\boldsymbol{I})}^{J} \boldsymbol{e}_{J} & \text { (no sum on } \boldsymbol{I}) \\
& =\frac{\boldsymbol{I} !}{\boldsymbol{I} !}|\varepsilon|_{\boldsymbol{I}}^{J} \boldsymbol{e}_{J} \quad \text { (using (A-34)) }
\end{array}
$$

It is concluded that

$$
\left(\iota_{S}\right)_{I}^{J}=|\varepsilon|_{I}^{J} .
$$

In addition, as the components of $T$ relative to the basis $\left\{\overleftarrow{\boldsymbol{e}}_{(I)}\right\}$ are $T^{\boldsymbol{I}}$

or

$$
\iota_{S}(T)=\left(\iota_{S}\right)_{\boldsymbol{I}}^{J} T^{\boldsymbol{I}} \boldsymbol{e}_{J}=|\varepsilon|_{I}^{J} T^{\boldsymbol{I}} \boldsymbol{e}_{J},
$$

$$
T^{J}=\left(\iota_{S}(T)\right)^{J}=|\varepsilon|_{I}^{J} T^{I},
$$


which could have been deduced otherwise.

Example 5. Consider the symmetrization mapping $\mathscr{Y}: \bigotimes^{l} \boldsymbol{V} \rightarrow \bigodot^{l} \boldsymbol{V}$. One has,

$$
\begin{aligned}
\mathscr{Y}\left(\boldsymbol{e}_{J}\right) & :=\boldsymbol{e}_{(J)} \\
& =|\varepsilon|_{J}^{\boldsymbol{I}} \boldsymbol{e}_{(\boldsymbol{I})} \quad(\text { only one } \boldsymbol{I}) \\
& =|\varepsilon|_{J}^{\boldsymbol{I}} \frac{\boldsymbol{I} !}{|\boldsymbol{I}| !} \overleftarrow{\boldsymbol{e}}_{(\boldsymbol{I})}
\end{aligned}
$$

and it follows from the definition of a matrix that

In addition,

$$
\mathscr{Y}_{J}^{\boldsymbol{I}}=\frac{\boldsymbol{I} !}{|\boldsymbol{I}| !}|\varepsilon|_{J}^{\boldsymbol{I}} .
$$

$$
\begin{aligned}
\mathscr{S}(T)^{\boldsymbol{I}}=\mathscr{Y}_{J}^{\boldsymbol{I}} T^{J} & =\frac{\boldsymbol{I} !}{|\boldsymbol{I}| !}|\varepsilon|_{J}^{\boldsymbol{I}} T^{J} \\
& =T^{(\boldsymbol{I})} \quad(\text { using (A-36)). }
\end{aligned}
$$

A.8. Duality. Consider the dual basis $\left\{\boldsymbol{e}^{i}\right\}$ of the dual vector space $\boldsymbol{V}^{*}$ so that $\boldsymbol{e}^{i}\left(\boldsymbol{e}_{j}\right)=\delta_{j}^{i}$. For any two multi-indices $I, J$, with $|I|=|J|=l$, we consider the action $\boldsymbol{e}^{(I)}\left(\boldsymbol{e}_{(J)}\right)$. We have

$$
\begin{aligned}
\boldsymbol{e}^{(I)}\left(\boldsymbol{e}_{(J)}\right) & =\left(\boldsymbol{e}^{i_{1}} \odot \cdots \odot \boldsymbol{e}^{i_{l}}\right)\left(\boldsymbol{e}_{j_{1}} \odot \cdots \odot \boldsymbol{e}_{j_{l}}\right) \\
& =\frac{1}{(l !)^{2}}\left(\sum_{p \in \mathscr{P}_{l}} \boldsymbol{e}^{i_{p(1)}} \otimes \cdots \otimes \boldsymbol{e}^{i_{p(l)}}\right)\left(\sum_{q \in \mathscr{P}_{l}} \boldsymbol{e}_{j_{q(1)}} \otimes \cdots \otimes \boldsymbol{e}_{j_{q(l)}}\right) \\
& =\frac{1}{(l !)^{2}} \sum_{p \in \mathscr{P}_{l}}\left(\sum_{q \in \mathscr{P}_{l}} \delta_{q(J)}^{p(I)}\right) \\
& =\frac{1}{(l !)^{2}} \sum_{p \in \mathscr{P}_{l}} I !|\varepsilon|_{J}^{p(I)} \quad \text { (using (A-34)) } \\
& =\frac{I !}{(l !)^{2}} \sum_{p \in \mathscr{P}_{l}}|\varepsilon|_{J}^{I} \quad \text { (thing (A-12)) } \\
& =\frac{I ! l !}{(l !)^{2}}|\varepsilon|_{J}^{I} \\
& =\frac{I !}{|I| !}|\varepsilon|_{J}^{I} .
\end{aligned}
$$

It follows from the identity above that for nondecreasing multi-indices $I, J$,

$$
\boldsymbol{e}^{(\boldsymbol{I})}\left(\overleftarrow{\boldsymbol{e}}_{(\boldsymbol{J})}\right)=\boldsymbol{e}^{(\boldsymbol{I})}\left(\frac{|\boldsymbol{J}| !}{\boldsymbol{J} !} \boldsymbol{e}_{(\boldsymbol{J})}\right)=|\varepsilon|_{J}^{\boldsymbol{I}}=\delta_{\boldsymbol{J}}^{\boldsymbol{I}}
$$

where one realizes that if the two multi-indices are nondecreasing, one can be a permutation of the other only when they are equal. 
The last identity implies that the basis $\left\{\boldsymbol{e}^{(\boldsymbol{I})}\right\}$ is the dual basis of $\left\{\overleftarrow{\boldsymbol{e}}_{(\boldsymbol{J})}\right\}$, and in particular,

$$
\bigodot^{l} \boldsymbol{V}^{*} \simeq\left(\bigodot^{l} \boldsymbol{V}\right)^{*}
$$

Finally, for $T=T^{\boldsymbol{I}} \overleftarrow{\boldsymbol{e}}_{(\boldsymbol{I})} \in \bigodot^{l} \boldsymbol{V}$ and $\psi=\psi_{\boldsymbol{I}} \boldsymbol{e}^{(\boldsymbol{I})} \in \bigodot^{l} \boldsymbol{V}^{*}$

$$
\psi(T)=\psi_{\boldsymbol{I}} T^{\boldsymbol{I}} .
$$

A.9. Symmetrization of cotensors and cosymmetrization. The inclusion of symmetric tensors in the collection of all tensors induces by duality a projection

$$
\iota_{S}^{*}:\left(\bigotimes^{l} \boldsymbol{V}\right)^{*} \simeq \bigotimes^{l} \boldsymbol{V}^{*} \rightarrow\left(\bigodot^{l} \boldsymbol{V}\right)^{*} \simeq \bigodot^{l} \boldsymbol{V}^{*},
$$

such that

$$
\iota_{S}^{*}(\varphi)(T)=\varphi(\iota(T)),
$$

for every symmetric tensor $T$. Thus, referring to elements of $\left(\otimes^{l} \boldsymbol{V}\right)^{*}$ as cotensors, $\iota_{S}^{*}$ is a symmetrization operator for cotensors.

One obtains

$$
\left(\iota_{S}^{*}(\varphi)\right)_{I}=\left(\iota_{S}^{*}\right)_{I}^{J} \varphi_{J}=|\varepsilon|_{I}^{J} \varphi_{J},
$$

where we observe that in the last expression one adds up the components of $\varphi$ corresponding to all permutations of $\boldsymbol{I}$, similarly to the symmetrization operation (but without taking the average).

In addition,

$$
\iota_{S}^{*}(\varphi)(T)=\left(\iota_{S}^{*}(\varphi)\right)_{I} T^{I}=|\varepsilon|_{I}^{J} \varphi_{J} T^{I}=\varphi_{J} T^{J},
$$

as expected. In the particular case where $\varphi$ is symmetric, using (A-32) and (A-72) gives

$$
\left(\iota_{S}^{*}(\varphi)\right)_{\boldsymbol{I}}=|\varepsilon|_{\boldsymbol{I}}^{J} \varphi_{J}=\frac{|\boldsymbol{I}| !}{\boldsymbol{I} !} \varphi_{\boldsymbol{I}},
$$

and

$$
\iota_{S}^{*}(\varphi)(T)=\varphi_{J} T^{J}=\sum_{\boldsymbol{I}} \frac{|\boldsymbol{I}| !}{\boldsymbol{I} !} \varphi_{\boldsymbol{I}} T^{\boldsymbol{I}} .
$$

The dual of the symmetrization mapping is (the cosymmetrization)

$$
\mathscr{\varphi}^{*}: \bigodot^{l} \boldsymbol{V}^{*} \rightarrow \bigotimes^{l} \boldsymbol{V}^{*}
$$

given by

$$
\mathscr{\varphi}^{*}(\psi)(T)=\psi(\mathscr{S}(T)) .
$$

Using the matrix obtained in Example 5, we have

$$
\mathscr{S}^{*}(\psi)(T)=\left(\mathscr{S}^{*}\right)_{J}^{\boldsymbol{I}} \psi_{\boldsymbol{I}} T^{J}=\sum_{\boldsymbol{I}, J} \frac{\boldsymbol{I} !}{|\boldsymbol{I}| !}|\varepsilon|_{J}^{\boldsymbol{I}} \psi_{\boldsymbol{I}} T^{J},
$$


and it follows that

$$
\mathscr{S}^{*}(\psi)_{J}=\sum_{\boldsymbol{I}} \frac{\boldsymbol{I} !}{|\boldsymbol{I}| !}|\varepsilon|_{J}^{\boldsymbol{I}} \psi_{\boldsymbol{I}}
$$

(It is observed that the sum over $\boldsymbol{I}$ contains only one nontrivial term.) In other words, if $J$ is a permutation of $\boldsymbol{I}$, then $\boldsymbol{I}=\langle J\rangle(\boldsymbol{I}$ is obtained by ordering $J)$, and

$$
\mathscr{S}^{*}(\psi)_{J}=\frac{\boldsymbol{I} !}{|\boldsymbol{I}| !} \psi_{\boldsymbol{I}}=\frac{J !}{|J| !} \psi_{\langle J\rangle} .
$$

In particular, if $T$ is symmetric, $\mathscr{S}^{*}(\psi)(T)=\psi(\mathscr{S}(T))=\psi(T)$, and so

$$
\sum_{J} \frac{J !}{|J| !} \psi_{\langle J\rangle} T^{J}=\sum_{I} \psi_{\boldsymbol{I}} T^{I}
$$

The last equation simply implies that for each nondecreasing $\boldsymbol{I}$ there are $|\boldsymbol{I}| ! / \boldsymbol{I} !=$ $|J| ! / J$ ! distinct indices $J$ obtained by permutations.

Setting

$$
\overleftarrow{T}^{I}:=\frac{|\boldsymbol{I}| !}{\boldsymbol{I} !} T^{\boldsymbol{I}}, \quad \stackrel{\rightarrow}{\rightarrow}^{K}:=\frac{K !}{|K| !} T^{K}
$$

one can write

$$
\psi_{\langle J\rangle} \stackrel{\vec{T}}{\rightarrow}^{J}=\sum_{I} \psi_{\boldsymbol{I}} T^{\boldsymbol{I}}, \quad \psi_{\langle J\rangle} T^{J}=\psi_{\boldsymbol{I}} \overleftarrow{\leftarrow}_{\leftarrow}^{\underline{I}}
$$

\section{References}

[Abraham et al. 1988] R. Abraham, J. E. Marsden, and T. Ratiu, Manifolds, tensor analysis, and applications, 2nd ed., Applied Mathematical Sciences 75, Springer, 1988.

[Comon et al. 2008] P. Comon, G. Golub, L.-H. Lim, and B. Mourrain, "Symmetric tensors and symmetric tensor rank", SIAM J. Matrix Anal. Appl. 30:3 (2008), 1254-1279.

[Greub 1978] W. Greub, Multilinear algebra, 2nd ed., Springer, 1978.

[Levi-Civita 1927] T. Levi-Civita, The absolute differential calculus (calculus of tensors), Blackie, London, 1927. Reprinted Dover, New York, 1977.

[Noll 1959] W. Noll, "The foundations of classical mechanics in the light of recent advances in continuum mechanics", pp. 266-281 in The axiomatic method, with special reference to geometry and physics (Berkeley, CA, 1957-1958), edited by P. S. L. Henkin and A. Tarski, North-Holland, Amsterdam, 1959.

[Palais 1968] R. S. Palais, Foundations of global non-linear analysis, W. A. Benjamin, New York, 1968.

[Saunders 1989] D. J. Saunders, The geometry of jet bundles, London Mathematical Society Lecture Note Series 142, Cambridge University Press, 1989.

[Segev 1986] R. Segev, "Forces and the existence of stresses in invariant continuum mechanics", $J$. Math. Phys. 27:1 (1986), 163-170.

[Segev 1994] R. Segev, "A geometrical framework for the statics of materials with microstructure", Math. Models Methods Appl. Sci. 4:6 (1994), 871-897.

[Segev 2000] R. Segev, "The geometry of Cauchy's fluxes", Arch. Ration. Mech. Anal. 154:3 (2000), 183-198. 
[Segev 2002] R. Segev, "Metric-independent analysis of the stress-energy tensor", J. Math. Phys. 43:6 (2002), 3220-3231.

[Segev 2013] R. Segev, "Notes on metric independent analysis of classical fields", Math. Methods Appl. Sci. 36:5 (2013), 497-566.

[Segev 2017] R. Segev, "Geometric analysis of hyper-stresses", Internat. J. Engrg. Sci. 120 (2017), $100-118$.

[Segev and Rodnay 1999] R. Segev and G. Rodnay, "Cauchy's theorem on manifolds", J. Elasticity 56:2 (1999), 129-144.

[Truesdell and Toupin 1960] C. Truesdell and R. Toupin, "The classical field theories", pp. 226-793; appendix, pp. 794-858 in Handbuch der Physik, vol. III/1, edited by S. Flügge, Springer, 1960.

Received 28 Oct 2017. Revised 8 Feb 2018. Accepted 21 Mar 2018.

REUVEN SEgEV: rsegev@bgu.ac.il

Department of Mechanical Engineering, Ben-Gurion University of the Negev, Beer-Sheva, Israel

JĘDRZEJ ŚNIATYCKI: sniatycki@gmail.com

Department of Mathematics and Statistics, University of Victoria, Victoria, BC, Canada and

Department of Mathematics and Statistics, University of Calgary, Calgary, AL, Canada 